\title{
Darkly Pigmented Melanophages Present
}

National Cancer Institute

\section{Source}

National Cancer Institute. Darkly Pigmented Melanophages Present. NCI Thesaurus. Code C96173.

A morphologic finding indicating the presence of darkly stained macrophages that contain melanin. 